ARTICLE

\title{
Commercial BWR Whole Core Calculations with MCNP5
}

\author{
Sho TAKANO ${ }^{1, *}$, Akiyuki TSUCHIYA ${ }^{2}$, Akiko TOISHIGAWA ${ }^{1}$, \\ Shingo FUJIMAKI ${ }^{1}$ and Tadashi IKEHARA ${ }^{1}$ \\ ${ }^{1}$ Global Nuclear Fuel-Japan, 3-1, Uchikawa 2-Chome, Yokosuka-Shi, Kanagawa-Ken 239-0836, JAPAN \\ ${ }^{2}$ Hitachi-GE Nuclear Energy, Ltd., 1-1, Saiwai-Cho 3-Chome, Hitachi-Shi, Ibaraki-Ken 317-0073, JAPAN
}

\begin{abstract}
Whole core critical calculations have been performed for a series of cold criticality experiments in the initial core of ABWR by using a continuous energy Monte Carlo code MCNP5. Three-dimensional geometrical models were precisely created to represent individual fuel rod inside fuel assemblies and absorber pin and plate inside control blades. The variance of calculated effective multiplication factor $k_{\text {eff }}$ from MCNP5 was within experimental errors. The observed bias of $k_{\text {eff }}$ from 1.0 was consistent with the known trend. Neutron history of $10^{8}$ was taken, the convergence of $k_{\text {eff }}$ and source distribution were checked by the sandwich method and the Shannon Entropy, respectively. Power distributions were evaluated at various levels of resolutions as fuel assembly, fuel rod and inside fuel rod, and the variances in those results were investigated. The results of $k_{\text {eff }}$ calculated for the cold criticality experiment were $1.0035(+/-0.1 \% \Delta \mathrm{k})$ with standard deviation less than $0.01 \% \Delta \mathrm{k}$, where the each computation time was approximately 12 hours on PC Linux server with duel Xeon quad $3.0 \mathrm{GHz}$ for 8 processors OpenMPI.
\end{abstract}

KEYWORDS: continuous energy Monte Carlo, MCNP5, commercial BWR, cold criticality experiments, $k_{\text {eff }}$, fuel node power, pin power, power within Gd-rod

\section{Introduction}

Continuous energy Monte Carlo codes are capable of simulating neutron transport phenomena inside a reactor core with explicit representations in geometry and material densities. Recent advances in PC based high performance computation technologies enable us to apply the Monte Carlo codes to large scale problems, such as detailed three-dimensional whole-core calculations for commercial power reactors within acceptable computation time at low cost, letting us to calculate the effective multiplication factor $k_{\text {eff }}$ along with the power distributions and the various design parameters by taking localized tallies by taking sufficient number of histories. ${ }^{1)}$

In this study, we applied MCNP5 to ABWR whole-core analyses to calculate $k_{\text {eff }}$ and the power distributions at multiple levels of geometrical scales, such as assembly averaged, fuel rod averaged and inner fuel rod power distributions. The calculation cases were chosen from a series of the measured cold criticality experiments, corresponding to various control rod patterns in the initial core loaded with 872 fresh $\mathrm{UO}_{2}$ fuel assemblies of $9 \times 9$ designs.

A difficulty in performing large scale Monte Carlo calculations is characterized as high in the dominance ratio (i.e., DR $~ 1.0$ ) of the problems, causing slower rate of convergence in the power iteration used for solving criticality calculations. A deterministic code AETNA, ${ }^{2)}$ a three-energy group core simulator based on the analytic polynomial nodal

*Corresponding author,E-mail: Shou.Takano@gnf.com

\footnotetext{
(C) 2011 Atomic Energy Society of Japan, All Rights Reserved.
}

method, was used to predetermine the value of DR for the initial cores of interest by calculating the eigenvalues of both the fundamental and the first higher mode. In the framework of Monte Carlo calculations, the fission matrix method and the coarse-mesh projection method are known for calculating the values of $\mathrm{DR},{ }^{3)}$ but they have not been incorporated into MCNP5 yet. To access the convergence of MCNP5 calculations, both $k_{e f f}$ and the Shannon entropy $H_{s r c}$ of source distributions ${ }^{4}$ ) were checked by taking the values of predetermined DR as a guiding parameter of the convergence.

Apart from the variance in the Monte Carlo calculation being proportional to $T^{-0.5}$, where $T$ is the total neutron histories, the bias in $k_{\text {eff }}$ was investigated by varying the number of the iterations under the constraint of keeping the total neutron histories constant. In addition, the behavior of convergence of $k_{\text {eff }}$ and $H_{s r c}$ was evaluated based on the sandwich method ${ }^{5)}$ by assuming the two types of initial source distributions which were uniform over the reactor core and localized at important region of the reactor cores.

Through the investigations mentioned in the above, it was concluded that the power iterations were converged without falling on fault convergences, when the order of 100 million histories with $M=20,000$ times $N=5,000$ are taken, where $M$ is the numbers of particles per cycle and $N$ is the number of the active cycles, respectively. The calculated $k_{\text {eff }}$ with their variances were compared with the results of the cold criticality experiments. The average power distributions for the fuel assembly and the average fuel rod quantities were compared with those from AETNA to evaluate the degree of the uncertainties in the power distributions which were cal- 
culated from the localized tallies. It was found that the calculated $k_{\text {eff }}$ values and the power distributions are satisfactory. Furthermore, the variances in the results of the inner fuel rod power distribution were investigated. Factor of five or more in $M$ is recommended to reduce the degrees of the uncertainties in the radial power distributions inside the fuel rods, especially for the Gd bearing rods.

To expand the realm of applications of MCNP5 from initial cores to those loaded with depleted fuel assemblies, MCNP5 needs to be enhanced to remove the upper limits on the numbers of surfaces and materials. In this study, the maximum numbers of the surface and the material cards specified are 86,000 and 8,900 , respectively, against the common maximum of 99,999 for the both. In addition, there are upper limits on the numbers of the cards specifying cells, universes and tallies.

By overcoming the limitations encountered when applying Monte Carlo codes to the problems having spatially large and complex geometry and as well as making efforts on porting these codes on high performance computation platforms, Monte Carlo codes will have a potential to become tools for obtaining the reference solutions in a commercial LWR core design analyses within acceptable computation time.

In this paper, the convergence of the power iterations is mainly discussed by paying attention on future applications of MCNP5 to depletion calculations.

\section{Calculation Method}

\section{Geometry}

The initial loading core of commercial ABWR, Shika-2, ${ }^{6}$ which consists of 872 fuel assemblies $(9 \times 9$, step3A) and 205 control rods, was evaluated. Both Boron Carbide control rods and Hafnium control rods were loaded. The enrichment of assembly type I is $1.5 \mathrm{wt} \%$ and that of assembly type II is $4.1 \mathrm{wt} \%$. The core average enrichment is $3.5 \mathrm{wt} \%$. The core height was approximately $3.7 \mathrm{~m}$ and the equivalent core diameter was $5.2 \mathrm{~m}$, respectively. The fuel loading pattern of 1st cycle for Shika-2 is shown in Fig. 1.

In calculations, fuel and moderator temperature were 293.15 K and the nuclear data used was ENDF/B-VII.0. Approximately $30 \mathrm{~cm}$ thick of water was added to upper and lower end of core. And also, the core was surrounded with approximately $1.5 \mathrm{~m}$ thick water. Outside of water, void surrounded the core. In the axial direction, the whole core was divided into 24 nodes correspond to enrichment and $\mathrm{Gd}_{2} \mathrm{O}_{3}$ distribution. The geometry was precisely modeled to represent the individual fuel pin and absorber pin and plate, except for the top position of control rod which was partially withdrawn. To simplify, we could only calculate the case in which the top position of control rod corresponded to axial node boundary. Therefore, when we compared node power distribution, we interpolated from two results. In other evaluation, we only calculated the case in which the top poison of control rod was the nearest to actual.

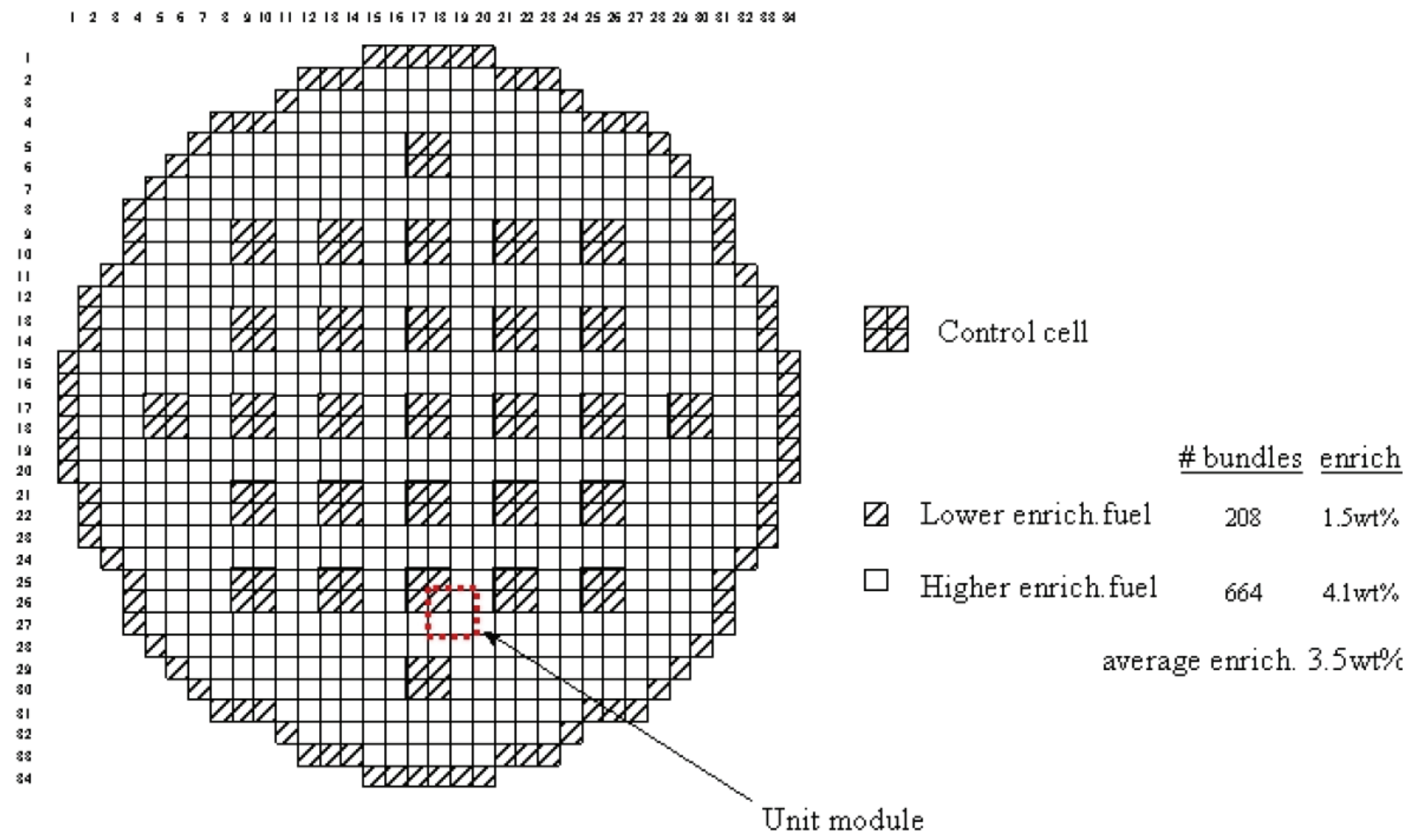

Fig. 1 Fuel loading pattern of 1 st cycle for Shika-2 

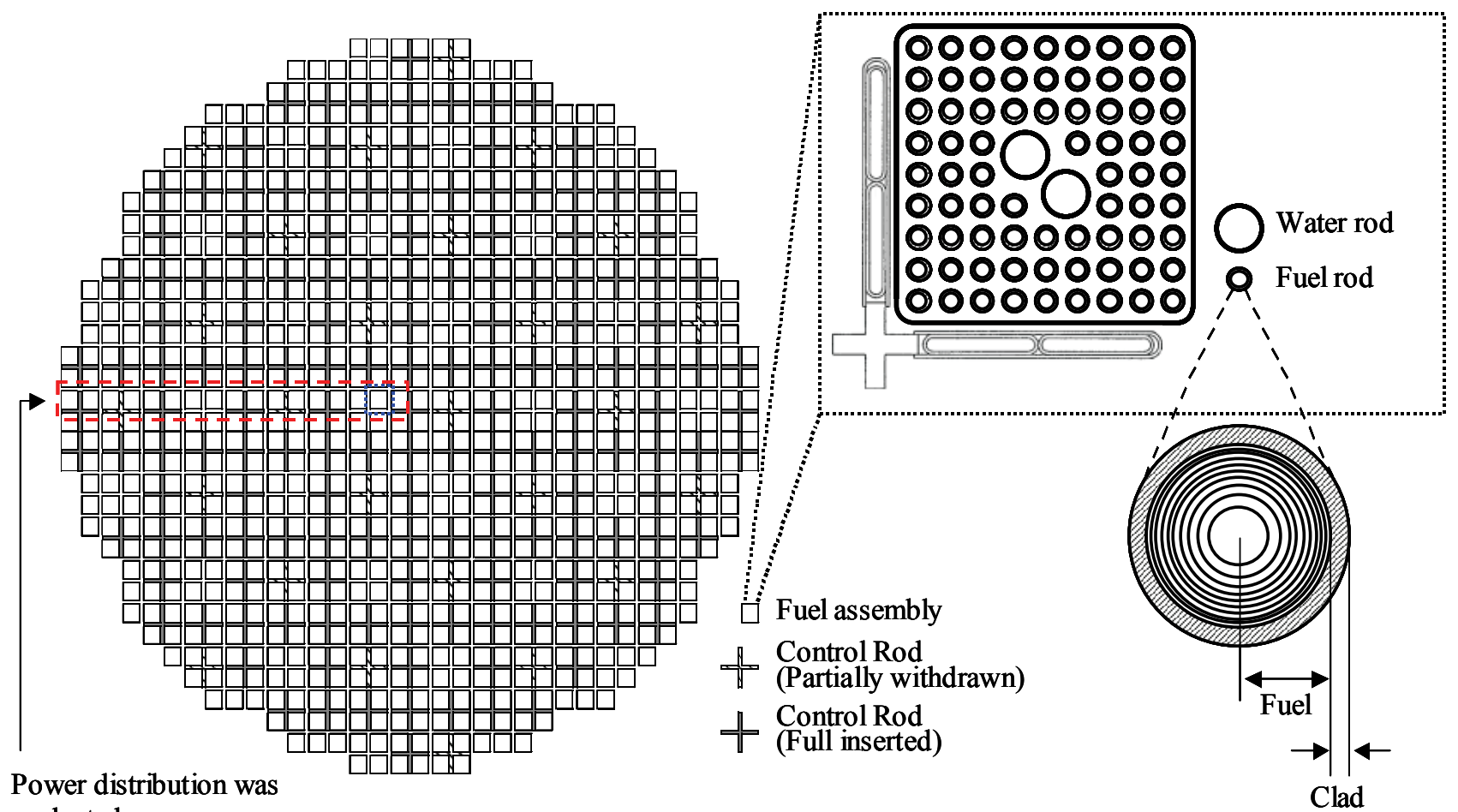

evaluated

Fig. 2 The sketch of evaluated core (first criticality test)
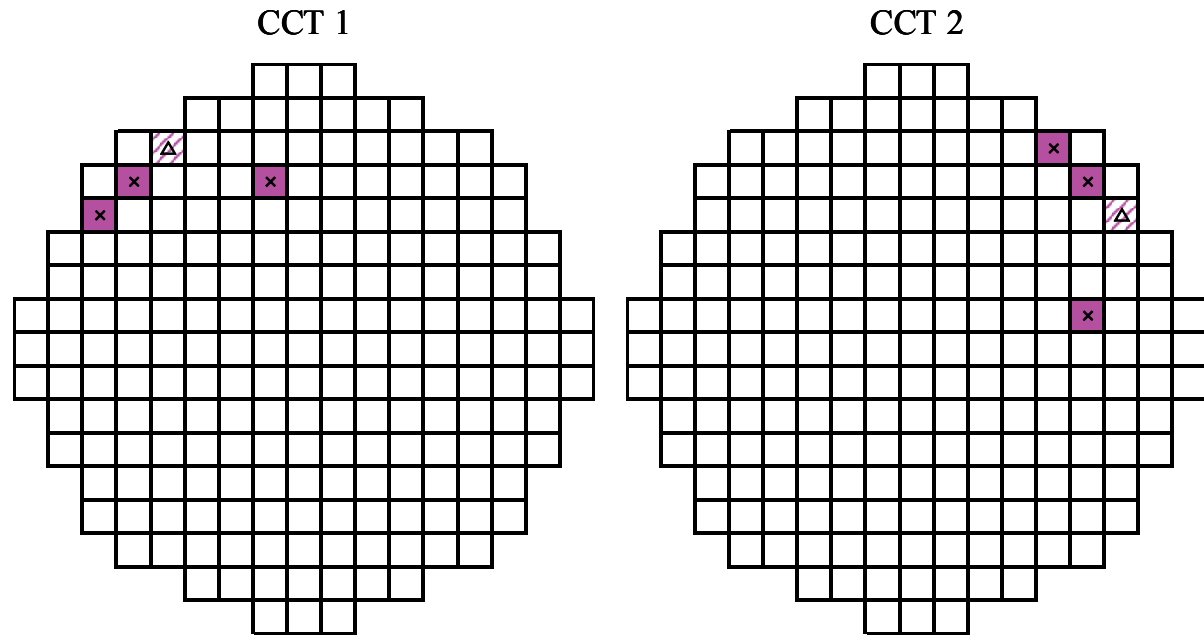

Full withdrawn

4 Partially withdrawn

Full inserted

Fig. 3 The control rod pattern of cold criticality test 1 and 2

The cold criticality experiments had different control rod insertion patterns, in which the control rods were withdrawn uniformly (first criticality test, called FCT) or some of the control rods were withdrawn locally (cold criticality test, called CCT). The sketch of FCT geometry and the control rod pattern of CCT are shown in Figs. 2 and 3, respectively. The specific Gd-rod was annularly divided into 10 spatial regions. In the $\mathrm{CCT}$, the locations of withdrawn control rods were different in each case except that CCT 1 - 4 were rotational symmetry. The cold criticality experiments were carried out with different temperature and period.

\section{Calculation Conditions}

Open MPI-based executable of MCNP5 was built to perform parallel computation on a PC Linux platform equipped with the dual Xeon quad core processors $(3.00 \mathrm{GHz})$ to realize OpenMPI parallel computation with eight processors at maximum on a single machine. A point-wise cross section library of MCNP5 (ACE library) is based on ENDF/B-VII.0.

Generally, a variance in a Monte Carlo result is proportional to $T^{0.5}$ where $T$ is number of total neutron particles. Furthermore, in order to achieve required accuracy, above a certain number of cycles was needed because of the differ- 


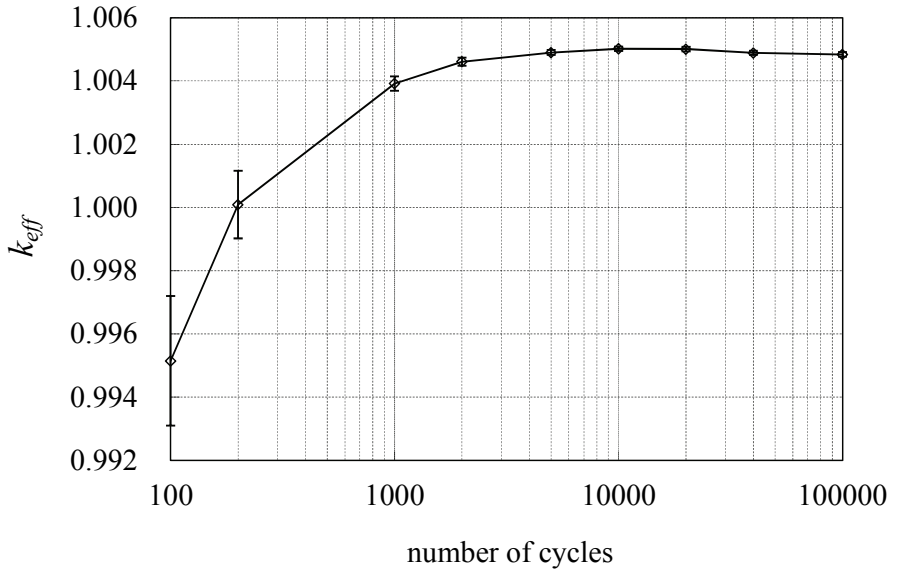

Fig. 4 Relation between $k_{\text {eff }}$ and the number of cycles

ence between initial source distribution and converged source distribution. The relation between the effective multiplication factor $k_{\text {eff }}$ and the number of cycles under conditions that total neutron particles was maintained constant $\left(10^{8}\right.$ particles $)$ and initial source was uniform distribution is shown in Fig. 4. This figure shows that over a few thousand cycles is required to attain $k_{\text {eff }}$ convergence. So we set the number of neutron particles per cycle to 20,000 and the number of active cycles to 5,000. According to our sensitivity study to the number of inactive cycle, the final $k_{\text {eff }}$ remain almost unchanged by taking it more than 100. $k_{\text {eff }}$ where inactive cycle was 100 agreed with $k_{\text {eff }}$ where inactive cycle was 1,000 within $0.01 \% \Delta \mathrm{k}$. Since over 100 inactive cycles did not affect the result, we set inactive cycle to 100 .

To take the case of FCT, the variance of $k_{\text {eff }}$ was less than $0.007 \% \Delta \mathrm{k}$ by tracking $10^{8}$ particles where the computation time was about 12 hours. By tallying the fuel node power and/or pin power, the computation time increased, e.g., it took about 1 day to tally all fuel node power.

\section{Result}

\section{The Effective Multiplication Factor $k_{e f f}$}

The effective multiplication factor $k_{\text {eff }}$ of cold criticality experiments were calculated. The results were shown in Fig. 5. These values were corrected for temperature and period reactivity individually by AETNA. The variances $(1 \sigma)$ of $k_{\text {eff }}$ from MCNP5 were less than $0.01 \% \Delta \mathrm{k}$ and the difference between maximum and minimum was about $0.13 \% \Delta \mathrm{k}$. The difference was larger than the variances from MCNP5. And also, there was difference among CCT 1 - 4 in which the locations of withdrawn control rods were rotational symmetry. The difference among CCT 1 - 4 corresponded approximately to experimental errors. It would appear that the experimental errors caused mainly the difference of $k_{\text {eff }}$. In other words, the $k_{\text {eff }}$ of cold criticality experiments obtained by MCNP5 agreed with each other within experimental errors. The observed bias of $k_{\text {eff }}$ from 1.0 was considered mainly arising from the uncertainty in the nuclear data, and consistent with the known trend of ENDF/B-VII.0, when employed to analyze cold, clean, $\mathrm{UO}_{2}$

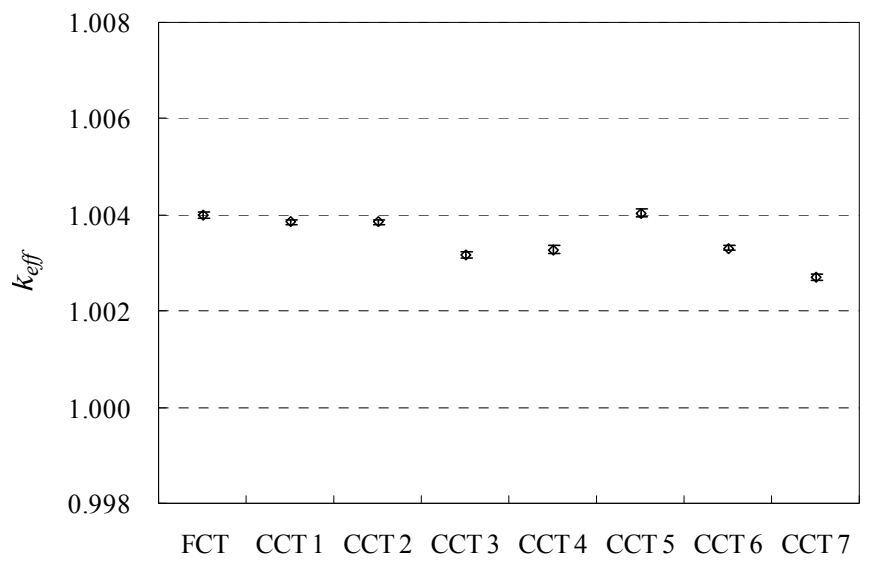

Fig. $5 k_{\text {eff }}$ of cold criticality experiments

critical experiments under LWR conditions. ${ }^{7)}$

\section{Convergence of The Effective Multiplication Factor $k_{\text {eff }}$}

The DR of cold criticality experiments were analyzed by 3 dimensional calculation code AETNA that is based on 3 group analytic-polynomial nodal expansion method. The DR of the FCT was largest among cold criticality experiments. So, we focused on FCT.

The variance in the estimate of $k_{\text {eff }}$ usually decreases as the total neutron particles increases. Even then, it is difficult to determine the final converged $k_{\text {eff }}$. In order to evaluate the convergence of $k_{\text {eff }}$, the sandwich method was applied to the FCT. The calculations were performed with two types of initial source, i.e., a uniform source and an important region source. Because the control rods were withdrawn uniformly, the important region is middle of core. We see from Fig. 6 that there is no significant difference between the results of $k_{\text {eff }}$ obtained from the uniform source and important region source. The result of the final $k_{\text {eff }}$ for the FCT was 1.0052 (without correction of temperature and period reactivity).

\section{Convergence of Source Distribution}

To evaluate the convergence of the fission source spatial distribution, MCNP5 computes a quantity called the Shannon entropy of fission source distribution, $H_{s r c}$. The Shannon entropy of the discretized source distribution is expressed by

$$
H_{s r c}=-\sum_{j=1}^{N_{\mathrm{s}}} P_{j} \cdot \ln _{2}\left(P_{j}\right),
$$

where $N_{s}$ is the number of grid boxes, and $P_{j}$ is the ratio of the number of source sites in $j$-th grid boxes to total number of source sites. The $H_{s r c}$ varies between 0 for a point source distribution to $\ln _{2}\left(N_{s}\right)$ for a uniform source distribution. Figure 7 shows the result of the Shannon entropy with grid boxes corresponds to fuel nodes. The calculations were performed with a uniform source and an important region source. If the final source distribution is uniform, $H_{s r c}$ 


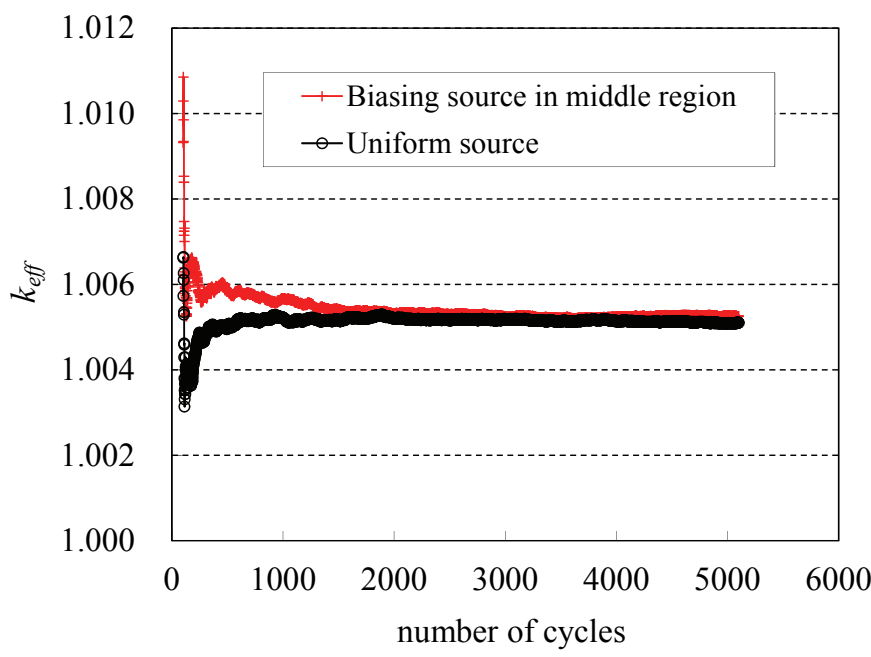

Fig. 6 Evaluation of $k_{\text {eff }}$ by using sandwich method with two initial source distribution

would be $14.8\left(=\ln _{2}(34 \times 34 \times 24)\right)$. We see from Fig. 7 that there is no significant difference between the source distribution obtained from the uniform source and important region source. Accordingly, tracking $10^{8}$ neutron particles is enough to achieve the convergence of source distribution.

\section{Fuel Node Power}

The fuel node power distribution appeared in Fig. 8. These values were shown for radial $\mathrm{x}$-direction from left boundary to the center. The radial y-coordinate is 17 th row and axial z-coordinate is 12 th node, that is, half the height of core. And then, these values were normalized by the average power of upper-left core region, where z-coordinate is 12 th node. Compared the node power distribution calculated by MCNP5 with that by AETNA, the difference was $2.3 \sigma$ on average, where $\sigma$ is variance in MCNP5. Although the largest difference was $5.0 \sigma$ except for lower power nodes, there was no specific tendency. Therefore, it is reasonable to conclude that MCNP5 was able to evaluate core characteristic

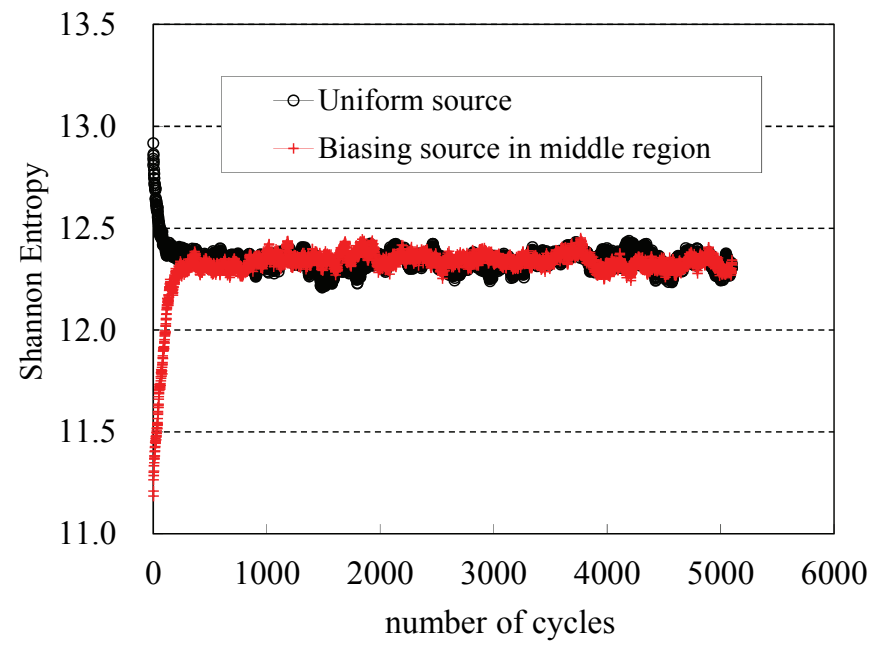

Fig. 7 Evaluation of Shannon Entropy with two initial source distribution

with satisfactory accuracy.

\section{Accuracy in Fuel Pin}

In this chapter, we focused on the fuel node of which relative power was one of the largest, i.e., $\mathrm{x}, \mathrm{y}, \mathrm{z}$-coordinate is 16 th, 17th, 12th node in the FCT. The control rod adjacent to the node given above was withdrawn.

To verify the pin power distribution, the infinite lattice calculation was performed. We set the number of neutron particles per cycle to 50,000 , the number of active cycles to 200 and the number of inactive cycles to 20 . The variances of the infinite lattice calculation are significantly small.

\section{Pin Power Distribution}

The fuel assembly consisted of $\mathrm{UO}_{2}$-rods, Gd-rods and water-rods. We evaluated the pin power distribution in the diagonal direction of lattice as shown in Fig. 9. The fuel pins were labeled from 1 st to 9 th. The location 3rd and 5th indicated Gd-rod and water-rod, respectively.

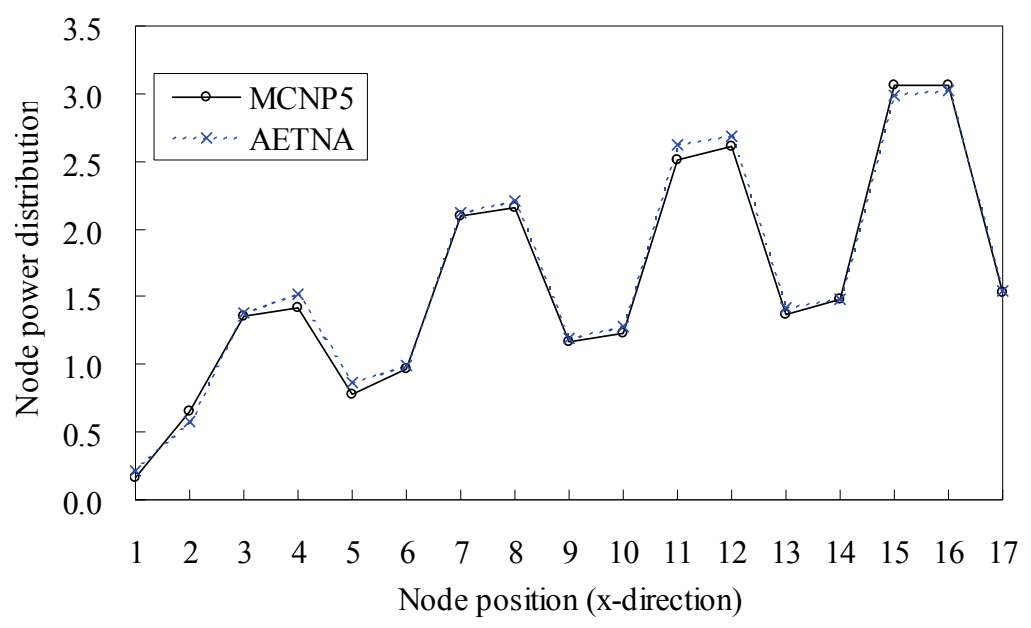

Fig. 8 Node power distribution (y,z-coordinate were 17th and 12th raw) 

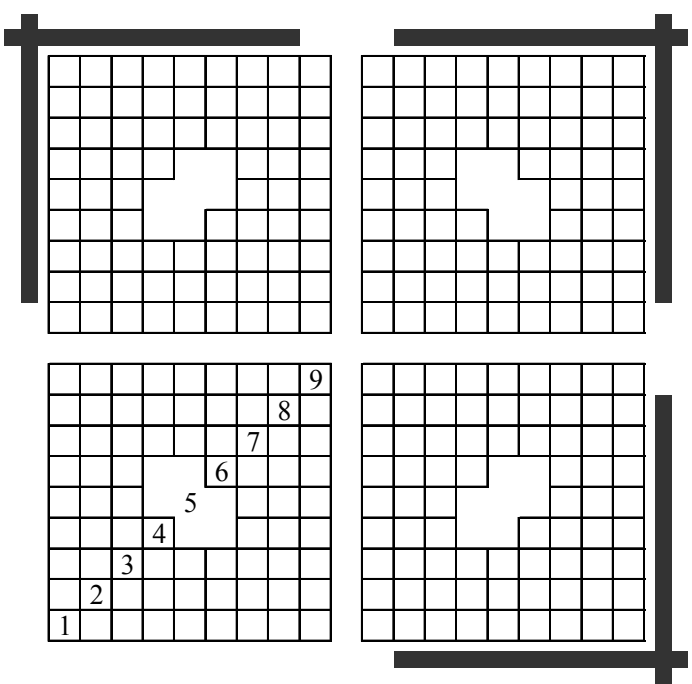

Fig. 9 Pin position in which power was evaluated $(\mathrm{x}, \mathrm{y}, \mathrm{z})=(16,17,12$ node $)$

The pin power distribution in the diagonal direction of lattice is shown in Fig. 10. Compared the result obtained from 100,000 neutron particles per cycle with that of the default calculation $(20,000$ particles per cycle), the difference is $0.9 \sigma$ on average. The largest difference is $2.8 \sigma$ in location 7 th, where the $1 \sigma$ was about 0.03 , and the difference is less than $2 \sigma$ in higher power pin. The default calculation seemed to be sufficient to achieve satisfactory accuracy. Moreover, the relative pin power distribution calculated by AETNA is quite similar to that by MCNP5. In the middle region, the pin power distribution by infinite lattice calculation with MCNP5 is also quite similar. On the contrary, there was difference that was not due to statistical uncertainty in the peripheral region. In the infinite lattice calculation, control rod was withdrawn. Because some control rods were inserted and others were withdrawn in the whole core calculation, the relative power was larger in the fuel pin closed to withdrawn control rod. In addition, the boundary condition of infinite lattice calculation was mirror reflection. Therefore, the pin power distribution by infinite lattice calculation did not correspond to the other results in peripheral region.

\section{Relative Power within Gd-Rod}

Within Gd-rod, Gd number density decreases from the surface to center region with exposure. In assembly calculation, it is the typical discretization method that Gd-rod is annularly divided into about 8 or 10 spatial regions due to the "onion-skin" effect."

The relative power distribution within the Gd-rod (location 3rd) was shown in Fig. 11. The Gd-rod was annularly divided into 10 spatial regions whose volume was equal to each other. The difference between the result obtained from 100,000 neutron particles per cycle and that of the default calculation $(20,000$ particles per cycle) is $0.5 \sigma$ on average. The largest difference is $1.1 \sigma$ in 9th ring from the center, where $1 \sigma$ is about 0.05 . The relative power at the surface neighbor region in the Gd-rod was larger than that at the center region. Although this tendency appeared in the result by default calculation $(20,000$ particles per cycle) on the whole, it was not necessary. The relative power in 4th ring from the center is smaller than that in 3rd ring. The result obtained from 100,000 neutron particles per cycle was more similar to the result of infinite lattice calculation.

\section{Matter to Calculate Fuel Pin Power}

As mentioned above, the default calculation $(20,000$ particles per cycle) seemed to be sufficient to achieve satisfactory accuracy in terms of pin power distribution. At least, we could also see that reasonable tendency appeared in the result of relative power distribution within Gd-rod by default calculation. But due to restriction of MCNP5 on total number of surfaces and so on, we could not evaluate power distribution within all Gd-rods under the present conditions. It was essential to improve MCNP5.

By assuming 3 items, we evaluated the computation time to apply MCNP5 to burn-up calculation. First, there was no

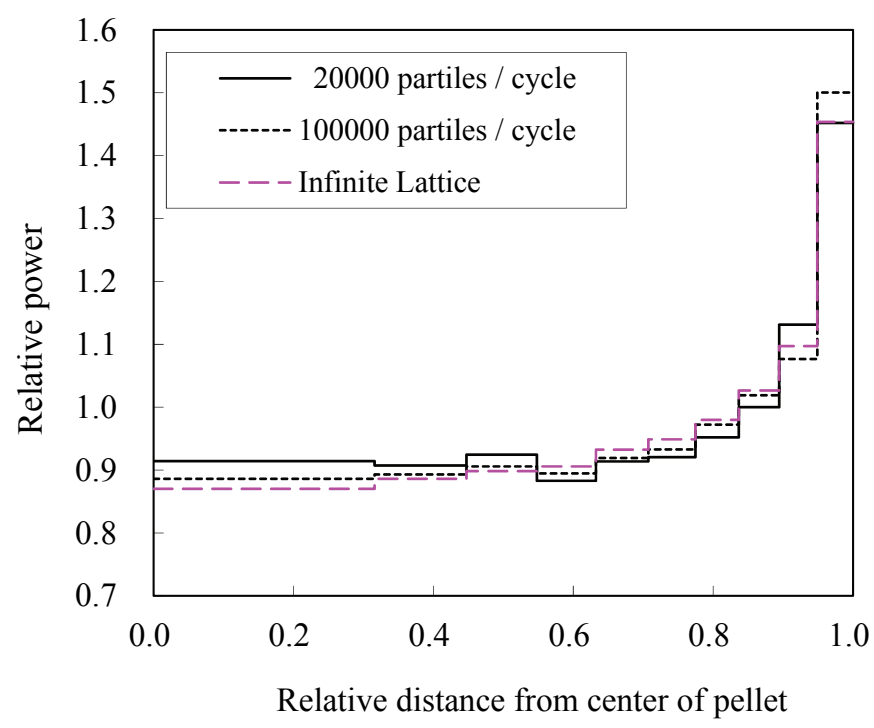

Fig. 11 Relative power distribution within Gd-rod 
restriction on the number of surfaces, materials and so on. Second, the result obtained from 100,000 neutron particles per cycle in whole core calculations was enough in terms of relative power within Gd-rod. Finally, the void iterations were ignored. At the moment, it took about a few days to tally the fuel pin power. The node peaking factor where relative node power was one of the largest was approximately 5 . Therefore, the computation time needed to run burn-up calculation may be over 1 month.

\section{Summary}

Whole core calculations have been performed for a commercial ABWR, Shika-2 by using MCNP5 with 20,000 neutron particles per cycle and 5,000 active cycles. The geometry of whole core was precisely modeled to represent the individual fuel pin and absorber pin and plate. The calculated $k_{\text {eff }}$ of cold criticality experiments agreed with each other within experimental errors. The observed bias of $k_{\text {eff }}$ from 1.0 was consistent with the known trend of ENDF/B-VII.0. Moreover, we confirmed the convergence of $k_{\text {eff }}$ and source distribution by means of the sandwich method and the Shannon Entropy, respectively. Node power distribution was in agreement with the result of AETNA based on deterministic theories. These results show that we are now able to evaluate core characteristics of commercial ABWR using MCNP5 with satisfactory accuracy.

The pin power distribution and the relative power distribution within Gd-rod were also evaluated by MCNP5. The pin power distribution was in good agreement with that of AENTA. We could also see reasonable relative power dis- tribution within Gd-rod. Assuming that there is no restriction of MCNP5 on total number of surfaces and materials, we can evaluate power distribution within all Gd-rods and apply MCNP5 to burn-up calculations.

\section{References}

1) M. Tohjoh et al., "Application of continuous-energy Monte Carlo code as a cross-section generator of BWR core calculations," Ann. Nucl.Energy., 32, 857 (2005).

2) T. Iwamoto et al., "Benchmarking and Applications of BWR Core Simulator AETNA," Advances in Nuclear Fuel Management III, Hilton Head Island, South Carolina (2003).

3) B. Nease et al., "Dominance ratio calculations with MCNP," International Conference on the Physics of Reactors, Interlaken, Switzerland, September 14-19 (2008).

4) X-5 Monte Carlo Team, MCNP - A General Monte Carlo $N$-Particle Transport Code, Version 5 Volume II User's Guide, LA-CP-03-0245, Los Alamos National Laboratory (LANL) (2003).

5) Y. Naito, J. Yang, "The sandwich Method for Determining Source Convergence in Monte Calro Calculation," J. Nucl. Sci. Tech., 41[5], 559 (2004).

6) F. Yoshimori, R. Masumi, "High-Burn-up Initial Core Concept SUMIT for BWRs and the results of its first application to Shika-2," 2005 Water Rector Fuel Perf. Mtg., Kyoto, Japan, Oct. 2-6, 2005 (2005).

7) T. Ikehara et al., "Analysis of Critical Experiments EPICURE, MISTRAL, BASALA and FUBILA with MCNP5," Proc. Spring Mtg. Of Atomic Energy Society of Japan, Osaka, Japan, Mar. 26-28, 2008 (2008), [in Japanese]. 